\title{
ON THE NUMBER OF FULLY WEIGHTED ZERO-SUM SUBSEQUENCES
}

\author{
ABÍLIO LEMOS, ALLAN O. MOURA, ANDERSON T. SILVA AND B. K. MORIYA
}

\begin{abstract}
Let $G$ be a finite additive abelian group with exponent $n$ and $S=g_{1} \cdots g_{t}$ be a sequence of elements in $G$. For any element $g$ of $G$ and $A \subseteq$ $\{1,2, \ldots, n-1\}$, let $N_{A, g}(S)$ denote the number of subsequences $T=\prod_{i \in I} g_{i}$ of $S$ such that $\sum_{i \in I} a_{i} g_{i}=g$, where $I \subseteq\{1, \ldots, t\}$ and $a_{i} \in A$. In this paper, we prove that $N_{A, 0}(S) \geq 2^{|S|-D_{A}(G)+1}$, when $A=\{1, \ldots, n-1\}$, where $D_{A}(G)$ is the smallest positive integer $l$, such that every sequence $S$ over $G$ of length at least $l$ has nonempty subsequence $T=\prod_{i \in I} g_{i}$ such that $\sum_{i \in I} a_{i} g_{i}=$ $0, I \subseteq\{1, \ldots, t\}$ and $a_{i} \in A$. Moreover, we classify the sequences such that $N_{A, 0}(S)=2^{|S|-D_{A}(G)+1}$, where the exponent of $G$ is an odd number.
\end{abstract}

\section{INTRODUCTION}

Let $G$ be a finite additive abelian group with exponent $n$ and $S$ be a sequence over $G$. The enumeration of subsequences with certain prescribed properties is a classical topic in Combinatorial Number Theory going back to Erdös, Ginzburg and Ziv (see [7, 13, 14]) who proved that $2 n-1$ is the smallest integer, such that every sequence $S$ over a cyclic group $C_{n}$ has a subsequence of length $n$ with zero-sum. This raises the problem of determining the smallest positive integer $l$, such that every sequence $S=g_{1} \cdots g_{l}$ has a nonempty zero-sum subsequence. Such an integer $l$ is called the Davenport constant of $G$ (see [6, 20]), denoted by $D(G)$, which is still unknown for wide class of groups. In an analogous manner, for a nonempty subset $A \subseteq \mathbb{Z} \backslash\{k n: k \in \mathbb{Z}\}$, Adhikari et. al. (see [1]) defined an $A$-weighted Davenport constant, denoted by $D_{A}(G)$, to be a smallest $t \in \mathbb{N}$ such that every sequence $S$ over $G$ of length $t$ has nonempty $A$-weighted zero-sum subsequence.

For any $g$ of $G$, let $N_{A, g}(S)$ (when $A=\{1\}$ we write $N_{g}(S)$ ) denote the number of weighted subsequences $T=\prod_{i \in I} g_{i}$ of $S=g_{1} \cdots g_{l}$ such that $\sum_{i \in I} a_{i} g_{i}=g$, where $I \subseteq\{1, \ldots, l\}$ is a nonempty subset and $a_{i} \in A$. In 1969, 21] proved that $N_{0}(S) \geq 2^{|S|-D(G)+1}$ for every sequence $S$ over $G$ of length $|S| \geq D(G)$. Subsequently, several authors, including [2, 3, 4, 8, 9, 10, 11, 12, 15, 16, 17, 18. obtained a huge variety of results on the number of subsequences with prescribed properties. Recently, Chang et al. [5] found the lower bound of $N_{g}(S)$ for any arbitrary $g$.

In this paper, we determine a lower bound for $N_{A, 0}(S)$, where $A=\{1,2, \ldots, n-1\}$ and $G$ is an additive finite abelian group with exponent $n$. We also characterize the structures of the extremal sequences which attain the lower bound for the group $G$, such that $n$ is an odd number.

2010 Mathematics Subject Classification. 20K01, $11 \mathrm{~B} 75$.

Key words and phrases. Finite abelian group, weighted subsequences; Davenport constant. 


\section{NotATIONS AND TERMinOlogies}

In this section, we will introduce some notations and terminologies. Let $\mathbb{N}_{0}$ be the set of non-negative integers. For integers $a, b \in \mathbb{N}_{0}$, we define $[a, b]=$ $\left\{x \in \mathbb{N}_{0}: a \leq x \leq b\right\}$.

For a sequence

$$
S=\prod_{i=1}^{m} g_{i} \in \mathcal{F}(G)
$$

where $\mathcal{F}(G)$ is the free abelian monoid with basis $G$, a subsequence $T=g_{i_{1} \ldots} g_{i_{k}}$ of $S$, with $I_{T}=\left\{i_{1}, \ldots, i_{k}\right\} \subseteq[1, m]$ is denoted by $T \mid S$; we identify two subsequences $S_{1}$ and $S_{2}$ if $I_{S_{1}}=I_{S_{2}}$. Given subsequences $S_{1}, \ldots, S_{r}$ of $S$, we define $\operatorname{gcd}\left(S_{1}, \ldots, S_{r}\right)$ to be the sequence indexed by $I_{S_{1}} \cap \cdots \cap I_{S_{r}}$. We say that two subsequences $S_{1}$ and $S_{2}$ are disjoint if $\operatorname{gcd}\left(S_{1}, S_{2}\right)=\lambda$, where $\lambda$ refers to the empty sequence. If $S_{1}$ and $S_{2}$ are disjoint, then we denote by $S_{1} S_{2}$ the subsequence with set index $I_{S_{1}} \cup I_{S_{2}}$; if $S_{1} \mid S_{2}$; we denote by $S_{2} S_{1}^{-1}$ the subsequence with set index $I_{S_{2}} \backslash I_{S_{1}}$.

Moreover, we define

(1) $|S|=m$ the length of $S$.

(2) an A-weighted sum is a sum of the form $\sigma^{\mathbf{a}}(S)=\sum_{i=1}^{m} a_{i} g_{i}$, with $\mathbf{a}=$ $a_{1} \cdots a_{m} \in \mathcal{F}(A)$, where $\mathcal{F}(A)$ is the free abelian monoid with basis $A$. When $A=[1, n-1]$, we call $S$ a fully weighted sequence.

(3) $\sum_{A}(S)=\left\{\sum_{i \in I} a_{i} g_{i}: \emptyset \neq I \subseteq[1, m]\right.$ and $\left.a_{i} \in A\right\}$, a set of nonempty $A$ weighted subsums of $S$.

According to the above definitions, we adopt the convention that $\sigma^{\mathbf{a}}(\lambda)=0$, for any $\mathbf{a} \in \mathcal{F}(A)$. For convenience, we define $\sum_{A}^{\bullet}(S)=\sum_{A}(S) \cup\{0\}$.

The sequence $S$ is called

(1) an $A$-weighted zero-sum free sequence if $0 \notin \sum_{A}(S)$,

(2) an $A$-weighted zero-sum sequence if $\sigma^{\mathbf{a}}(S)=0$ for some $\mathbf{a} \in \mathcal{F}(A)$.

For an element $g \in G$, let

$$
N_{A, g}(S)=\left|\left\{I \subseteq[1, m]: \sum_{i \in I} a_{i} g_{i}=g, a_{i} \in A\right\}\right|
$$

denote the number of subsequences $T$ of $S$ with $\sigma^{\mathbf{a}}(T)=g$ for some $\mathbf{a} \in \mathcal{F}(A)$.

Definition 2.1. Let $n$ be the exponent of $G, g \in G, A \subseteq \mathbb{Z} \backslash\{k n: k \in \mathbb{Z}\}$ and $S \in \mathcal{F}(G)$. We say $S$ is g-complete sequence with weight in $A$ if $N_{A, g}(S) \geq$ $2^{|S|-D_{A}(G)+1}$. We call $S$ an extremal $g$-complete sequence with respect to $A$ if $N_{A, g}(S)=2^{|S|-D_{A}(G)+1}$. Let us denote $C_{A, g}(\mathcal{F}(G))$ as the set of all $g$-complete sequences with respect to $A$ and $E C_{A, g}(\mathcal{F}(G))$ as the set of all extremal $g$-complete sequences with respect to $A$.

Definition 2.2. Let $n$ be the exponent of $G$ and $A \subseteq \mathbb{Z} \backslash\{k n: k \in \mathbb{Z}\}$. We say $G$ is a 0-complete group with respect to $A$ if $\mathcal{F}(G)=C_{A, 0}(\mathcal{F}(G))$.

When $A=\{1\}$, Olson [21] proved that all finite abelian groups are 0-complete with respect to $A$. Chang et al. [5] proved, that, when $A=\{1\}$, if $g \in \sum_{A}^{\bullet}(S)$, then $S \in C_{A, g}(\mathcal{F}(G))$ and, if $S$ is extremal $h$-complete sequence with respect to $A$ for some $h \in G$, then $S$ is $g$-complete sequence with respect to $A$ for all $g \in G$. Moreover, they classified the sequences in $E C_{A, 0}(\mathcal{F}(G))$ to $G$ with odd order. 
Here we present an example: Take an $A$-weighted zero-sum free sequence $U$ over $G$ with $|U|=D_{A}(G)-1$. Thus, for $S=U 0^{|S|-D_{A}(G)+1}$ and for all $g \in \sum_{A}^{\bullet}(U)$, we have $S \in C_{A, g}(\mathcal{F}(G))$ and $S \in E C_{A, 0}(\mathcal{F}(G))$.

\section{LOWER BOUND}

We write the finite abelian group $G$ as direct sum $G=H \oplus C_{n}^{r}$, where $C_{n}^{r}$ denotes $r$ copies of the cyclic group of order $n$ denoted by $C_{n}$ and $H=C_{n_{1}} \oplus \cdots \oplus C_{n_{t}}$ with $1<n_{1}\left|n_{2}\right| \cdots\left|n_{t}\right| n=\exp (G)$ and $n_{t}<n$.

To prove our first theorem, we need some auxiliary results, which are as follows.

Proposition 3.1. [Proposition 2.3 [19] Let $G$ be a finite abelian group with exponent $n, A \subseteq[1, n-1]$ a nonempty subset and $b \in \mathbb{N}$. Then,

$$
D_{b A}(G)=D_{A}(\operatorname{gcd}(b, n) G) .
$$

Corollary 3.2. Let $G$ be a finite abelian group with exponent $n$ and $A \subseteq[1, n-1]$ be a nonempty subset, such that $A \cup\{0\}$ is a proper subgroup of $\mathbb{Z}_{n}$. Then, $D_{A}(G)=$ $D_{A^{\prime}}(d G)$, where $A=d A^{\prime}$ and $A^{\prime} \cup\{0\} \cong \mathbb{Z}_{n / d}$.

Proof. Since $A \cup\{0\}$ is a proper subgroup of $\mathbb{Z}_{n}$, then there is $d \in \mathbb{Z}_{n}$ such that $A \cup$ $\{0\}=\langle d\rangle$ and $d$ is the minimum with this property. We observe that $d=\operatorname{gcd}(d, n)$. Now, we apply the Proposition 3.1 with $b=d$ and obtain $D_{A}(G)=D_{A^{\prime}}(d G)$, where $A=d A^{\prime}$. Since $d \mathbb{Z}_{n / d}=d\left\{0,1,2, \ldots, \frac{n}{d}-1\right\} \cong\langle d\rangle=A \cup\{0\}=d\left(A^{\prime} \cup\{0\}\right)$, then $A^{\prime} \cup\{0\} \cong \mathbb{Z}_{n / d}$.

An immediate consequence of the Corollary 3.2 is that we could consider only fully weight instead of a proper subgroup.

Lemma 3.3. [Theorem 5.2 [19] Let $G=H \oplus C_{n}^{r}$, where $H=C_{n_{1}} \oplus \cdots \oplus C_{n_{t}}$ with $1<n_{1}\left|n_{2}\right| \cdots\left|n_{t}\right| n=\exp (G)$ and $n_{t}<n$. Then, $D_{A}(G)=r+1$.

A subsequence $T$ of $S$ is called a maximal A-weighted zero-sum free subsequence if $T$ is a subsequence of maximal length such that $T$ is $A$-weighted zero-sum free.

Below, we present an important result for the fully weighted Davenport constant.

Theorem 3.4. All finite abelian group $G$ with exponent $n$ is 0 -complete with respect to $A=[1, n-1]$.

Proof. According to Lemma 3.3, we can write $D_{A}(G)=r+1$. If $|S| \leq r$, then $N_{A, 0}(S) \geq 1 \geq 2^{|S|-r}$. If $|S|=r+1$, then there is an $A$-weighted zero-sum nonempty subsequence $T$ of $S$. Thus, $N_{A, 0}(S) \geq 2=2^{|S|-r}$. Notice that if there is no $T \mid S$, such that $T$ is no a maximal $A$-weighted zero-sum free with $|T|=r$, then $N_{A, 0}(S)>2^{|S|-r}$.

Suppose now $r+1<|S|$.

We divide the proof in three cases:

Case 1: Let $S \in \mathcal{F}(G)$ be a sequence such that $o(g)<n$ for all $g \mid S$ :

In this case, we have that each $g \mid S$ is an $A$-weighted zero-sum subsequence and so $N_{A, 0}(S)=2^{|S|}>2^{|S|-r}$.

Case 2: Let $S=T W \in \mathcal{F}(G)$ be a sequence such that the elements of $T$ have order $n$ and $T$ is maximal $A$-weighted zero-sum free with $|T|<r$.

Then, for each element $g \mid W$, we have two possibilities:

a) If $o(g)<n$ and for $a_{g}=o(g) \in A$, then $g$ is an $A$-weighted zero-sum subsequence. 
b) If $o(g)=n$, then $T g$ has an $A$-weighted zero-sum subsequence with $g$ being one of its elements.

In both possibilities, there is $V \mid T g$, such that $V$ is an $A$-weighted zero-sum subsequence whose coefficient of $g$ is $a_{g} \in A$. Then, $a_{g} g$ is an $A$-weighted sum of some subsequence of $T$ :

$$
a_{g} g=\sum_{i \in I_{g}} a_{i} g_{i} ; I_{g} \subset I_{T}
$$

Thus, for every $U \mid W$ nonempty, we have

$$
\sum_{g \mid U} a_{g} g=\sum_{g \mid U} \sum_{i \in I_{g}} a_{i} g_{i}=\sum_{g_{i} \in I_{V}} b_{i} g_{i} ; I_{V} \subset I_{T}, \text { with } a_{i}, b_{i} \in A,
$$

i.e., the $A$-weighted sum $\sum_{g \mid U} a_{g} g$ is an $A$-weighted sum of some subsequence $V_{U}$ of $T$. Therefore, $U V_{U}$ is an $A$-weighted zero-sum subsequence of $S$. Notice that if $V_{U}=\lambda$, then $U$ is an $A$-weighted zero-sum subsequence. Therefore, if we include the empty subsequence, we obtain a minimum of $2^{|W|}=2^{|S|-|T|}$ distinct $A$-weighted zero-sum subsequences of $S$. This proves that $N_{A, 0}(S)>2^{|S|-r}$.

Case 3: Let $S=T W \in \mathcal{F}(G)$ be a sequence such that $T$ is a maximal $A$ weighted zero-sum free and $|T|=r$.

As in Case 2, we obtain a minimum of $2^{|W|}=2^{|S|-|T|}$ distinct $A$-weighted zerosum subsequences of $S$. Therefore, $N_{A, 0}(S) \geq 2^{|S|-r}$.

\section{The structures of eXtremal Sequences on the Fully Weighted DAVENPORT CONSTANT}

In this section, we consider that $G$ is of the form $H \oplus C_{n}^{r}$ with $\exp (H)<n$ and we will study sequence $S$, such that $N_{A, 0}(S)=2^{|S|-D_{A}(G)+1}$ where $A=[1, n-1]$. The case $A=\{1\}$, for the general group of odd exponent, was studied by Chang et al. [5].

Because $N_{A, 0}(S)=2 N_{A, 0}\left(S 0^{-1}\right)$ and if $o(g)<n$, then $N_{A, 0}(S)=2 N_{A, 0}\left(S g^{-1}\right)$, it suffices to consider sequences $S$, such that $0 \nmid S$ and $o(g)=n$ for all $g \mid S$.

Proposition 4.1. Let $G$ be a finite abelian group with $\exp (G)=n$. If $S \in$ $E C_{A, g}(\mathcal{F}(G))$, with $A=[1, n-1], 0 \nmid S$ and $o(g)=n$ for all $g \mid S$, then $r \leq|S|$ and there is $T=\prod_{i=1}^{r} g_{i}$ a maximal A-weighted zero-sum free, such that

$$
S=\prod_{i=1}^{r} g_{i} \prod_{j=1}^{k} h_{j},
$$

where $k \in \mathbb{N}_{0}$ and $b_{j} h_{j}=\sum_{i \in I_{j}} a_{i} g_{i}$ with $a_{i}, b_{j} \in A, I_{j} \subset[1, r]$.

Proof. Let $S$ be a sequence over $G$, with $0 \nmid S, o(g)=n$ for all $g \mid S$ and $N_{A, 0}(S)=$ $2^{|S|-D_{A}(G)+1}=2^{|S|-r}$. If $|S|<r$, then as $\lambda$ is an $A$-weighted zero-sum subsequence of $S$ and $N_{A, 0}(S) \geq 1>2^{|S|-r}$. Therefore, we can assume $|S| \geq r$. By Theorem 3.4. Case 3, there is $T \mid S$, such that $T=\prod_{i=1}^{r} g_{i}$ is a maximal $A$-weighted zero-sum free, otherwise $N_{A, 0}(S)>2^{|S|-r}$. According to Lemma 3.3. we observe that if $g \nmid T$ then $a_{l} g=\sum_{i \in I \subset[1, r]} a_{i} g_{i}$, with $a_{i}, a_{l} \in A$. If there is more than a set $I$ satisfying this, then by Theorem 3.4. Case 3, it follows that $N_{A, 0}(S)>2^{|S|-r}$. Therefore, there is only one set $I$ satisfying $a_{l} g=\sum_{i \in I \subset[1, r]} a_{i} g_{i}$. Thus, we conclude that $S=\prod_{i=1}^{r} g_{i} \prod_{j=1}^{k} h_{j}$, where $b_{j} h_{j}=\sum_{i \in I_{j}} a_{i} g_{i}$ with $a_{i}, b_{j} \in A, I_{j} \subset[1, r]$.

Now, we prove the main result of this section. 
Theorem 4.2. Let $G$ be a finite abelian group with $\exp (G)=n$ an odd number. If $S \in E C_{A, g}(\mathcal{F}(G))$, with $A=[1, n-1], 0 \nmid S$ and $o(g)=n$ for all $g \mid S$, then $r \leq|S| \leq 2 r$ and there is $T=\prod_{i=1}^{r} g_{i}$ a maximal $A$-weighted zero-sum free, such that

$$
S=\prod_{i=1}^{r} g_{i} \prod_{j=1}^{k} h_{j},
$$

where $k \in[1, r], b_{j} h_{j}=\sum_{i \in I_{j}} a_{i} g_{i}$ with $a_{i}, b_{j} \in A, I_{j} \subset[1, r]$ and $I_{j}^{\prime} s$ are pairwise disjoint $\left(I_{j}=\emptyset\right.$ for all $j$ implies that $\left.S=\prod_{i=1}^{r} g_{i}\right)$.

Proof. Let $S$ be a sequence over $G$ with $0 \nmid S, o(g)=n$ for all $g \mid S$ and $N_{A, 0}(S)=$ $2^{|S|-D_{A}(G)+1}=2^{|S|-r}$. We know, by Proposition 4.1, that $S=\prod_{i=1}^{r} g_{i} \prod_{j=1}^{k} h_{j}$ where $k \in \mathbb{N}_{0}, b_{j} h_{j}=\sum_{i \in I_{j}} a_{i} g_{i}$ with $a_{i}, b_{j} \in A, I_{j} \subset[1, r]$ and $T=\prod_{i=1}^{r} g_{i}$ is a maximal $A$-weighted zero-sum free.

Now, we will prove that the $I_{j}^{\prime} s$ are pairwise disjoint. If $|S|=D_{A}(G)-1=r$, then $N_{A, 0}(S)=1, I_{j}=\emptyset$ for all $j \in[1, k]$ and $S=\prod_{i=1}^{r} g_{i}$. Suppose that $|S|=D_{A}(G)=r+1$ then, $I_{j} \neq \emptyset$ for only one $j, N_{A, 0}(S)=2$ and $S=\prod_{i=1}^{r} g_{i} h_{j}$. Finally, suppose $S=\prod_{i=1}^{r} g_{i} \prod_{j=1}^{k} h_{j}$ with $k \geq 2$ and $I_{j_{1}} \cap I_{j_{2}} \neq \emptyset$ for some $j_{1}, j_{2} \in[1, k]$, with $j_{1} \neq j_{2}$ and where

$$
a_{j_{1}} h_{j_{1}}=\sum_{i \in I_{j_{1}}} a_{i} g_{i} \text { and } a_{j_{2}} h_{j_{2}}=\sum_{i \in I_{j_{2}}} b_{i} g_{i}
$$

with $a_{j_{1}}, a_{j_{2}}, a_{i}, b_{i} \in A$.

Using the same argument of Theorem 3.4 Case 3, we have $\left(\begin{array}{l}k \\ 0\end{array}\right)+\left(\begin{array}{l}k \\ 1\end{array}\right)+\cdots+\left(\begin{array}{l}k \\ k\end{array}\right)=$ $2^{k}=2^{|S|-r} A$-weighted zero-sum subsequences of $S$. Since $I_{j_{1}} \cap I_{j_{2}} \neq \emptyset$, we have $I_{x}, I_{y} \subset I_{j_{1}} \cup I_{j_{2}}$ such that

$$
a_{j_{1}} h_{j_{1}}+a_{j_{2}} h_{j_{2}}=\sum_{i \in I_{x}} c_{i} g_{i} \text { and } a_{j_{1}} h_{j_{1}}-a_{j_{2}} h_{j_{2}}=\sum_{i \in I_{y}} d_{i} g_{i}, c_{i}, d_{i} \in A .
$$

Notice that $\exp (G)=n$ is an odd number, it follows that $I_{x} \neq \emptyset$ or $I_{y} \neq \emptyset$.

If $I_{x} \neq I_{y}$, then there is a new $A$-weighted zero-sum subsequence of $S$ and therefore $N_{A, 0}(S)>2^{|S|-r}$, which is a contradiction. Now, suppose that $I_{x}=I_{y}$ and take $g_{l} \mid \prod_{i \in I_{j_{1}} \cap I_{j_{2}}} g_{i}$ (observe that $c_{l} \neq 0, d_{l} \neq 0$ in (4.3)). Consider $T g_{l}^{-1}=$ $\prod_{i=1}^{r+1} g_{i} g_{l}^{-1}$, where $g_{r+1}=h_{j_{2}}$. If $T$ is not a maximal $A$-weighted zero-sum free, then there is $\bar{I}_{j_{2}} \subset[1, \ldots, r+1] \backslash\{l\}$ such that $z_{j_{2}} h_{j_{2}}=\sum_{i \in \bar{I}_{j_{2}}} s_{i} g_{i}$, i.e., we can obtain a new $A$-weighted zero-sum subsequence of $S$ and thus $N_{A, 0}(S)>2^{|S|-r}$, which is a contradiction. If $T$ is a maximal $A$-weighted zero-sum free, then by Lemma 3.3 we have $\bar{I}_{j_{1}} \subset[1, \ldots, r+1] \backslash\{l\}$ such that $v_{j_{1}} h_{j_{1}}=\sum_{i \in \bar{I}_{j_{1}}} u_{i} g_{i}$, i.e., we can obtain a new $A$-weighted zero-sum subsequence of $S$. Therefore, we have $N_{A, 0}(S)>2^{|S|-r}$ again, which is a contradiction.

We observe that if $k>r$, then there are $I_{j_{1}}$ and $I_{j_{2}}$ with $j_{1} \neq j_{2}$, such that $I_{j_{1}} \cap I_{j_{2}} \neq \emptyset$. Therefore, $N_{A, 0}(S)>2^{|S|-r}$. Thus, $r \leq|S| \leq 2 r$.

Because of the aforementioned, we make the following conjecture:

Conjecture 4.3. All finite abelian group $G$ with exponent $n$ is 0 -complete with respect to $A \subseteq \mathbb{Z} \backslash\{k n: k \in \mathbb{Z}\}$. 


\section{ACKNOWLEDGEMENT}

We would like to thank referees for all suggestions.

\section{REFERENCES}

[1] S. D. Adhikari, Y.G Chen, J. B. Friedlander, S. V. Konyagin, F. Pappalardi, Contributions to zero-sum problems, Discrete Math. 306 (2006), no. 1, 1-10.

[2] E. Balandraud, An addition theorem and maximal zero-sum free set in Z/pZ, Israel J. Math. 188 (2012), 405-429.

[3] A. Bialostocki and M. Lotspeich, Some developments of the Erdös-Ginzburg-Ziv Theorem I, Sets, Graphs and Numbers, Coll. Math. Soc. J. Bolyai 60 (1992), 97-117.

[4] H.Q. Cao and Z.W. Sun, On the number of zero-sum subsequences, Discrete Math. 307 (2007), 1687-1691.

[5] G. J. Chang, S. Chen, Y. Qu, G. Wang, H. Zhang, On the number of subsequence with a give sum in finite abelian group, Electron. J. Combin. 18 (2011), no. 1, paper 133.

[6] H. Davenport, On the addition of residue classes, J. Lond. Math. Soc. 10 (1935), 30-32.

[7] P. Erdös, A. Ginzburg, A. Ziv, Theorem in the additive number theory, Bulletim Research Council Israel 10F, 41-43, 1961.

[8] Z. Füredi and D.J. Kleitman, The minimal number of zero sums, Combinatorics, Paul Erdös is Eighty, J. Bolyai Math. Soc. (1993), 159-172.

[9] W.D. Gao, On the number of zero-sum subsequences, Discrete Math. 163 (1997), 267-273.

[10] W.D. Gao, On the number of subsequences with given sum, Discrete Math. 195 (1999), $127-138$.

[11] W.D. Gao and A. Geroldinger, On the number of subsequences with given sum of sequences over finite abelian p-groups, Rocky Mountain J. Math. 37 (2007), 1541- 1550.

[12] W.D. Gao and J.T. Peng, On the number of zero-sum subsequences of restricted size, Integers 9 (2009), 537-554.

[13] A. Geroldinger and F. Halter-Koch, Non-unique factorizations, Combinatorial and Analytic Theory, Pure and Applied Mathematics 278, Chapman \& Hall/CRC, 2006.

[14] A. Geroldinger, Additive group theory and non-unique factorizations, Combinatorial Number Theory and Additive Group Theory, Advanced Courses in Mathematics, CRM Barcelona, Birkhauser, (2009), 1-86.

[15] D.J. Grynkiewicz, On the number of m-term zero-sum subsequences, Acta Arith. 121 (2006), $275-298$.

[16] D.J. Grynkiewicz, E. Marchan and O. Ordaz, Representation of finite abelian group elements by subsequence sums, J. Theor. Nombres Bordeaux 21 (2009), 559-587.

[17] D.R. Guichard, Two theorems on the addition residue classes, Discrete Math. 81 (1990), $11-18$.

[18] M. Kisin, The number of zero sums modulo $\mathrm{m}$ in a sequence of length $\mathrm{n}$, Mathematica 41 (1994), 149-163.

[19] L. E. Marchan, O. Ordaz and W. A. Schmid, Remarks on the plus-minus weighted Davenport constant, International Journal of Number Theory 10 (05) (2014), 1219-1239.

[20] J. E. Olson. A combinatorial problem on finite abelian groups I, Jornal of Number Theory $\mathbf{1}$ (1969), 8-10.

[21] J. E. Olson. A combinatorial problem on finite abelian groups II, Jornal of Number Theory 1 (1969), 195-199.

Departamento de Matemática, Universidade Federal de Viçosa, Viçosa-MG, Brazil

E-mail address: abiliolemos@ufv.br, allan.moura@ufv.br, anderson.tiago@ufv.br

E-mail address: bhavinkumar@ufv.br 